\title{
Rectal Evacuatory Dysfunction:An Overview of Causes, Consequences and Cures
}

\section{Bandipalyam V Praveen*}

Department of Surgery, Southend University Hospital, West Cliff on Sea, Essex SSO ORY, UK

${ }^{*}$ Corresponding author: Bandipalyam V Praveen, Department of Surgery, Southend University Hospital, West Cliff on Sea, Essex SSO ORY, UK, Telephone: +1702 435555; E-mail: bandipalyam.praveen@southend.nhs.uk

Rec date: March 13, 2017; Acc date: March 17, 2017; Pub date: March 20, 2017

Citation: Praveen BV. Rectal Evacuatory Dysfunction: An Overview of Causes, Consequences and Cures. J Clin Gastroenterol Hepatol $2017,1: 1$.

\section{Introduction}

Evacuatory Dysfunction is "difficulty in emptying the rectum which may occur even with frequent visits to the toilet and/ or passing soft motions ". An alternative definition for evacuatory dysfunction is "a constellation of symptoms such as prolonged repeated straining at bowel movements, sensation of incomplete evacuation, and the need for digital manipulation".

Defaecation is a complex process involving integration of somatic and visceral muscle function with sensory information under local, spinal and central control. A random sample, in the United States adult population, showed that $4.6 \%$ had symptoms of outlet obstruction. Studies on care-home residents done by us showed symptoms of evacuatory dysfunction in more than half of the residents. Evacuatory dysfunction has significant effects on QoL as well as major financial implications for the health service.

\section{Causes of Rectal Evacuatory Dysfunction (RED)}

The patho-physiology of RED is shown in Table $\mathbf{1}$ and the various causes are listed in Table 2 . It is important to remember the various other non-bowel factors are also important such as age, BMI, associated co-morbidities, medications, hysterectomy, diet, psychiatric conditions, neurogenic and bowel operations amongst others.

Table 1: Patho-physiology of rectal evacuatory dysfunction.

\begin{tabular}{|l|l|}
\hline S. No & Patho-physiology of RED \\
\hline 1 & Mechanical \\
\hline 2 & Functional \\
\hline 3 & Neurogenic \\
\hline 4 & Systemic causes \\
\hline 5 & Post-Surgery \\
\hline 6 & Mixed \\
\hline
\end{tabular}

The mechanical causes mainly relate to situations where the normal anatomy of the ano-rectum is distorted or displaced. This could relate to prolapse of bowel which can be either internal (Rectal intussusceptions) or external (overt mucosal or full thickness rectal prolapsed), pockets which can develop, either anteriorly (rectocoele) and posteriorly, as well alterations in ano-rectal angle and indentation of the wall (enterocoele). The mechanical consequences can be the cause or effect of a neurogenic problem. A primary rectal hyposensitivity can lead to increased rectal loading, development of megarectum with increased rectal pressures and consequent secondary mechanical consequences. Alternatively, the mechanical issues can lead to a stretch neuropathy with additional problems.

The pathophysiology of pelvic dys-synergia in causation of evacuatory dysfunction is described in Figure 1.

The classic comparison of rectal evacuation can be made with getting toothpaste out of the tooth-paste tube (Figure 2).

The 3 crucial requirements common to both drive home the message. The first requirement is relaxation of the anal sphincter comparable to the removal of the cap from the tube. The second requirement would be the rectal contraction equivalent to squeezing the tooth-paste tube. The third one is the need for the tooth-paste to be present in adequate amounts and consistency to come out easily - which equates with the need for stools with optimum consistency to have been delivered to the rectum.

Another equally useful comparison to describe the role of the different groups of muscles during evacuation is to describe it in terms of a 'doubles tennis match'. This would consist of a team of two players 'serving' and the other team of two players 'receiving'. During evacuation, there are a team 'squeezing '(abdominal and rectal) and a team 'relaxing' (pelvic floor and anal sphincters).

The neurogenic cause may lie anywhere along the 'neural pathway' as indicated in Table 2.

The end results of any neurogenic lesion in the ano-rectal neural pathway can be on the sensory or motor function or both - with expected consequences. The reduced rectal sensation (Hyposensitivity) will lead to increased rectal loading, megarectum, incomplete emptying due to both inadequate feedback and deranged local reflexes. These can lead to secondary consequences like Rectocoele and Rectal intussusceptions and tertiary problems like urgency, faecal incontinence and haemorrhoids. 
Various systemic factors such as Systemic Sclerosis, dementia, Ehlers Danlos syndrome, learning disabilities, and medications can affect the evacuation process. These factors act at various levels - higher centers, stool consistency or the muscles and supporting tissues.

Table 2: Causes of rectal evacuatory dysfunction.

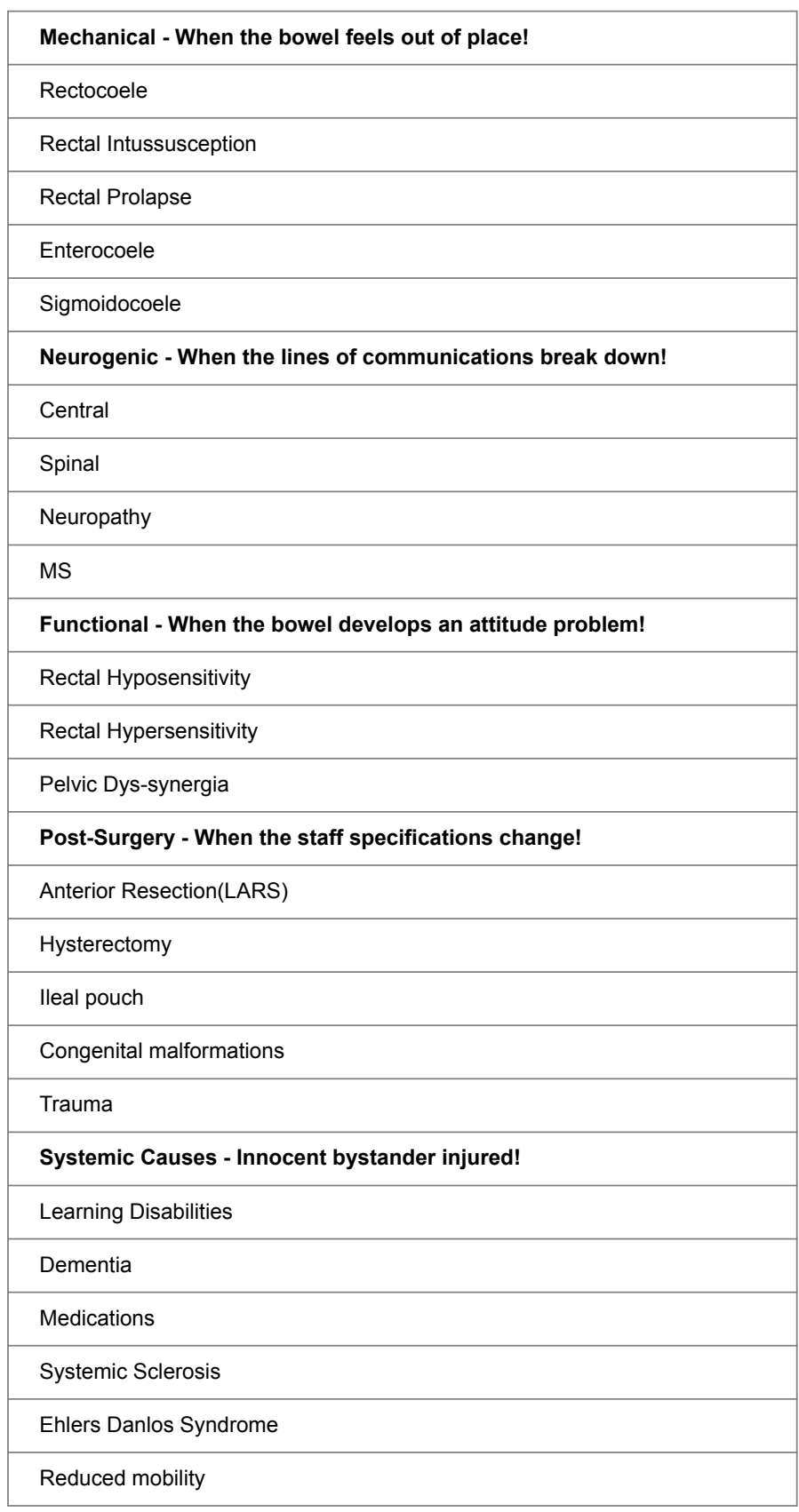

The post-surgery conditions following operations such as anterior resection, ileal pouch, hysterectomy and other pelvic procedures. These can affect the evacuatory function in various ways - by distorting the anatomy, reducing capacity, affecting compliance, causing nerve disruptions, changing stool consistency and reducing stool transit time. The effects of these operations should be anticipated and the patients appropriately consented/counselled prior to the primary bowel operations which predispose to RED. The symptoms of RED should be actively sought post-surgery by administering validated questionnaires which can help in planning treatments for the symptomatic patients.

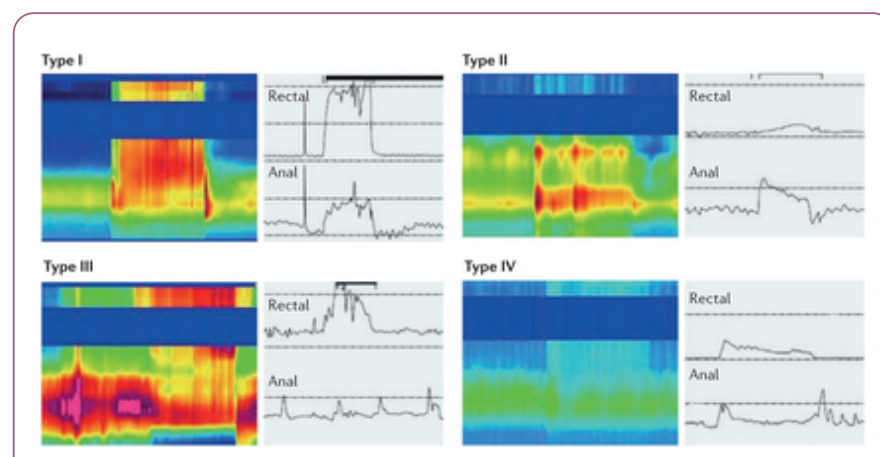

Figure 1: Types of pelvic dyssynergia, Type I: Adequate rectal push effort with paradoxical anal sphincter contraction, Type II: Inadequate rectal push effort with paradoxical anal sphincter contraction, Type III: Adequate rectal push effort but inadequate relaxation $(<20 \%)$ of anal sphincter pressure, Type IV: Inadequate rectal push effort and also inadequate relaxation $(<20 \%)$ of anal sphincter pressure.

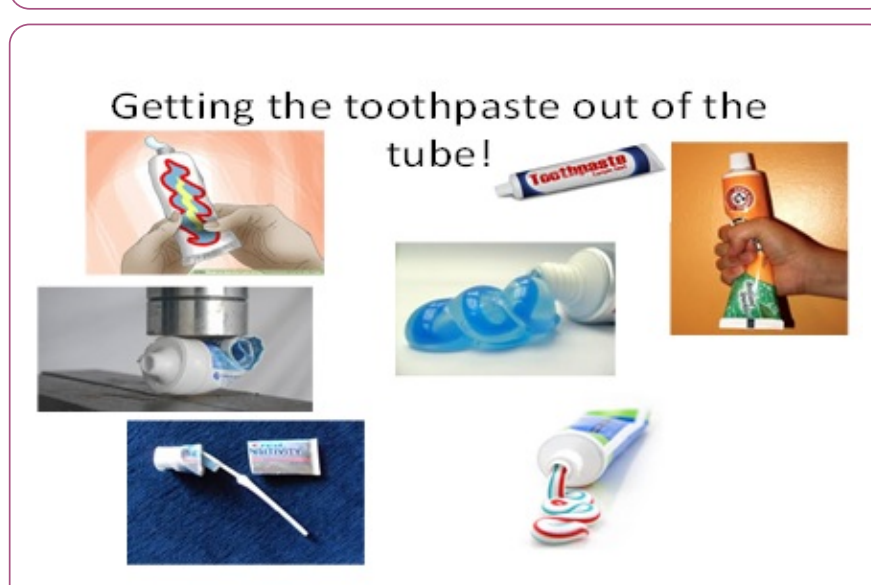

Figure 2: Pelvic dys-synergia mechanism is explained.

Not uncommonly, the evacuatory problem is a result of multiple issues from the various groups. The relative roles of the different factor should be analysed so that the most effective treatment can be considered.

\section{Consequences of Rectal Evacuatory Dysfunction}

The natural course of the pelvic floor dysfunction is associated with progressive deterioration leading to further consequences, which may become presenting symptoms. For example, the primary problem of rectal hyposensitivity can lead to issues such as Megarectum and changes in rectal compliance. The presentation can then be symptoms of lack of rectal filling sensation and incomplete emptying. This can lead to secondary mechanical consequences such as Rectal Intussusception and Rectocoele. The symptoms can then reflect these and include sensation of blockage, vaginal bulge/ 
pressure. The incomplete emptying can lead to urgency and urge leakage or post defaecation/passive leakage. Further progression of the condition can lead to tertiary consequences such as haemorrhoids, anal fissure, anal skin tags which may be the presenting complaints.

The symptoms of rectal bleeding or signs of Solitary Rectal Ulcer Syndrome (SRUS) may also create anxieties of any underlying sinister cause. Other consequences are dyspareunia, chronic pelvic pain or bladder symptoms. These are also likely to impact on all aspects of quality of life personal, social and sexual. The patient is likely to become more and more housebound and detaching themselves from most activities. Finally reduced mobility, depression and low self-esteem may result.

\section{Cures and Symptom Improvement Measures in Rectal Evacuatory Dysfunction}

\section{Broad principles of management}

It is to be remembered that these problems have a long natural course which are influenced by multiple factors. It is hence sensible to identify the most important symptom, so that priorities can be decided. The treatment options for the most important symptom can then be discussed to address the patient's 'felt need'. It is important that the expectations are realistic. MDT discussions should be held as per local guidelines. It is important to make it very clear to the patient that there are no quick fix solutions or guaranteed solutions. The role of surgery should be discussed and emphasized that surgery is only required in a minority of patients. This is planned only after all non-surgical treatments are tried. The natural course is one of gradual deterioration and hence there is a chance of recurrence even after surgery.

\section{Role of investigations}

Many objective tests are presently available which can support the decision-making process. Evacuatory Proctogram is still the 'gold standard' to evaluate the anorectal emptying. MR Proctogram is an alternative, which can avoid radiation in the younger patient. The proctogram can help with various information including rectal capacity, rectal sensation, anorectal angles during different states, completeness and time taken for emptying, mechanical issies like rectocoele, rectal intussusceptions, enterocoele as well as functional issues such as dyssynergia.

The anorectal manometry is a useful investigation to help know about the functional competence of anal sphincters, rectal sensation, Recto Anal Inhibitory Reflex (RAIR), length of anal canal and dyssnergia.

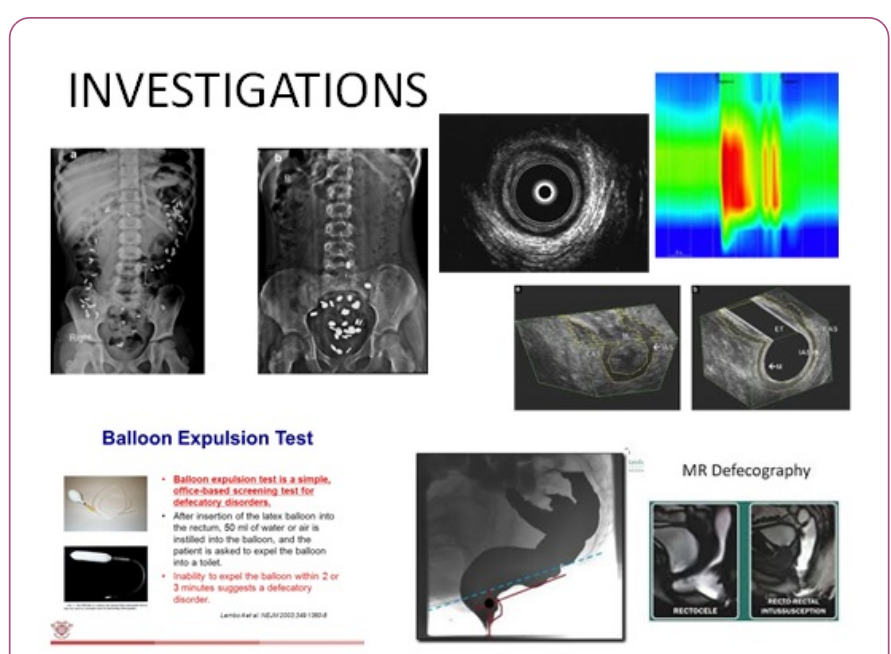

Figure 3: Investigations in rectal evacuatory dysfunction.

The endo-anal ultrasound helps with information about the structural integrity of the anal sphincters, associated conditions such as fistula, sepsis/collections amongst others.

The crucial thing is to ensure that the findings in the investigations are clinically relevant. It is important that the clinical symptoms, clinical signs and the investigation findings are triangulated to arrive at meaningful conclusions that can be helpful in making management decisions.

Apart from helping with diagnosis these investigations have an important role in various situations such as recording baseline parameters, research, audit, evaluating results of treatments, comparing treatments and medico-legal issues.

It is important that there is a well-defined clinical question before requesting any investigation - such a practice will ensure that investigations are done for a purpose and remain cost-effective Figure 3.

\section{Non-Surgical treatments}

Pelvic physiotherapy: This is usually one of the first steps in the management of any patient with pelvic floor dysfunction. The purpose is usually two fold - increase the tone/strength and improve co-ordination. This also combines education regarding defaecation techniques and may help to clarify many questions the patients have regarding their evacuation.

Bio-feedback: This would be an extension of the above physiotherapy - giving a feed-back to the patient regarding their own body processes. This better understanding leads to more conscious control and efforts to improve the defaecation dynamics. There are several methods used to provide this feedback - manometry, Electromyogram (EMG) and ultrasound. This can be useful in cases of rectal hyposensitivity, rectal hypersensitivity and pelvic dyssynergia.

Trans-anal irrigation: This can be effective when suppositories/enemas have not been helpful. The symptoms can be reduced sufficiently in order to avoid operations in many patients. This aims to give back control over the bowels 
back to the patients - who can choose the time and place at their convenience. It is safe and has good patient compliance. Good patient selection and tailored nurse training is the key to success. This can be administered in various ways - High volume (approx $700 \mathrm{ml}$ ) and Low volume $(120 \mathrm{ml})$. In the long run, this is cost-effective and has an established role in management of evacuatory dysfunction.

Neuro-modulation: Whilst this may not have a role in ODS, it can be part of the treatment in mixed cases where faecal leakage/associated sphincter incompetence is also present. A percutaneous method (PTNS) or Sacral Nerve Stimulation (SNS) may be used.

\section{Surgical treatments}

These are mainly useful in the management of Obstructed Defaecation Syndrome (ODS) due to mechanical issues such as Rectocoele, Enterocoele, Rectal Intussusception and Rectal Prolapse.

A laparoscopic approach is used in Lap VMR which involves a mesh to support the rectum and hitch it in place. The mesh can be associated with a small risk of infection or erosion in a small percentage of patients which makes clear pre-op consenting mandatory.
An alternative is a perineal approach and a trans-anal resection (STARR procedure) is done for intussusceptions and Altemeier's procedure for full thickness overt prolapse. A Delorme's procedure is nowadays only reserved for mucosal prolapse.

A rectocoele may also require surgery if non-surgical measures are not effective in controlling symptoms. A transvaginal approach is usually used.

\section{Conclusion}

Evacuatory Dysfunction is essentially a life-style issue. Earlier diagnosis is facilitated by increased awareness amongst both patients and clinicians. These patients include a heterogenous group of symptoms and conditions. They are best managed in specialist units in a MDT setting where diagnostic physiology investigations and effective treatments are available. Most patients do not require surgery and are best managed by non-surgical measures. Improving Quality of Life (QoL) is an important aspect of all treatment measures. 